Check for updates

Cite this: RSC Adv., 2019, 9, 7795

Received 4th December 2018

Accepted 26th February 2019

DOI: $10.1039 / \mathrm{c} 8 \mathrm{ra0} 9961 \mathrm{~h}$

rsc.li/rsc-advances

\section{The study of cationic waterborne polyurethanes modified by two different forms of polydimethylsiloxane}

\begin{abstract}
Qing Yu, Peiting Pan, Zongliang Du, Xiaosheng Du, (D) Haibo Wang (D) and Xu Cheng*
Two kinds of dimethylpolysiloxane, KF-6001 and X-22-176-DX, were used to modify polyurethane. The effects of KF-6001 and X-22-176-DX on the colloidal, physico-chemical and surface properties were studied for polydimethylsiloxane modified cationic waterborne polyurethanes (SiCWPUs). The chemical structures and the surface morphologies of the SiCWPUs are characterized via Fourier transform infrared spectrometry and scanning electron microscopy. The results showed that the addition of siloxane changes the structure and surface morphology of the polyurethane. The element distributions in the polymer films were tested via X-ray photoelectron spectroscopy, and the effect of the hydrophobicity of the surfaces of the polymer films of the cationic waterborne polyurethanes was demonstrated via water contact angle tests on the surfaces of the films. As the amount of siloxane added increases, the silicon content on the surfaces of the SiCPWU1 films increases from $0 \%$ to $17.92 \%$, and the actual silicon content on the surfaces of the films was much larger than the theoretical value. Therefore, the hydrophobicity of the membrane surface increases sharply, and the contact angle increases from $63.0^{\circ}$ to $105.3^{\circ}$. Dynamic mechanical analysis indicates that the introduction of polydimethylsiloxane into the cationic aqueous polyurethane chain increases microphase separation in the polymer films. Stress-strain data showed that the mechanical properties of SiCPWU1 films were better than those of SiCPWU2 films when the same amounts of PDMS were added.
\end{abstract}

\section{Introduction}

Waterborne polyurethanes (WPUs) have been increasingly used in coatings, elastomers, and other materials, because they are environmentally friendly and show high strength, high elasticity, and versatile performance. ${ }^{1-4}$ At present, a commonly used method is to introduce PEG or DMPA into polymers to prepare waterborne polyurethanes, but there has been little research into cationic waterborne polyurethanes (CWPUs) ${ }^{\mathbf{5 , 6}}$. CWPUs exhibit many unique characteristics, such as availability under acidic conditions, adhesion properties, antibacterial properties, and dustproof effects through electrostatic repulsion. ${ }^{7-10}$ These excellent characteristics give CWPUs the great potential to become high value-added materials. ${ }^{11,12}$ Nevertheless, the introduction of cationic groups into polyurethane segments could decrease the water resistance and surface hydrophobicity of WPU films. Therefore, most research has been focused on improving the performance of waterborne polyurethanes. ${ }^{13-15}$

Polydimethylsiloxane (PDMS) has unique properties, such as a low glass-transition temperature, low surface energy, good hydrophobicity, weather resistance, high flexibility and great

Textile Institute, College of Light Industry, Textile and Food Engineering, Sichuan University, Chengdu,610065, China.E-mail:scuchx@163.com thermal stability. ${ }^{\mathbf{1 6 , 1 7}}$ The incorporation of PDMS into polyurethane segments has been a frequently-used and effective method to improve the physical and chemical properties of WPUs. ${ }^{18-20} \mathrm{Xu}$ et al. $^{21}$ prepared a blocking polyether silicone oil with an amino group modified CWPU, which was used as the finishing agent for cashmere knitted fabric. Li et $a .^{22}$ researched the effects of the $\mathrm{N}$-methyldiethanolamine content, the $\mathrm{NCO} / \mathrm{OH}$ ratio, the trimethylolpropane content and other factors on the properties of polydimethylsiloxane polymers with monohydroxy modified cationic WPUs. A polydimethylsiloxane end-capped CWPU and polydimethylsiloxane with an amino group modified CWPU have been studied. However, most studies introduced PDMS into the main chain of the polyurethane, and little research has been concerned with polyurethane with PDMS as a side chain. The long PDMS segment in the form of a side chain could easily migrate to the surface.

In this work, in order to compare the effects of the form of polydimethylsiloxane on the polyurethane properties, two kinds of PDMS with dihydroxy groups were used to modify cationic waterborne polyurethane. And then, the effects of different structures of silicone on the performance of cationic waterborne polyurethanes were studied. The structures of the modified polyurethanes were characterized via Fourier transform infrared (FT-IR) spectroscopy. The microstructures, 
mechanical properties, thermal stabilities and surface properties of the polyurethane films were also compared in detail.

\section{Experimental}

\section{Materials}

Polypropylene glycol with a molecular weight of 2000 (PPG2000), purchased from Dragon Clan. Co. Ltd (Fujian, China), was placed under vacuum at $120^{\circ} \mathrm{C}$ for $2 \mathrm{~h}$ before use. Dibutyltin dilaurate (DBTDL) and isophorone isocyanate (IPDI) were acquired from Wanhua Polyurethane. Co. Ltd (Yantai, China). PDMS (materials: KF-6001, $M_{\mathrm{n}}=1800 \mathrm{~g} \mathrm{~mol}^{-1}$; and X-22-176-DX, $M_{\mathrm{n}}=3600 \mathrm{~g}$ $\mathrm{mol}^{-1}$ ) was supplied by Shin-Etsu Chemical Industry Co. Ltd. (Tokyo, Japan). The chemical structures of the PDMS samples used are shown in Scheme 1. $\mathrm{N}$-Methyl diethanolamine (MDEA) was provided by Huaxia Chemical Industry Co. (Chengdu, China). Acetic acid (HAc) and 1,4-butanediol (BDO) were purchased from Kelong Reagent Co. (Chengdu, China). MDEA and BDO were dried under vacuum at $80^{\circ} \mathrm{C}$ for $2 \mathrm{~h}$ before use. Deionized water was self-made in our laboratory.

\section{Instrumentation}

Fourier-transformed infrared (FTIR) spectra were collected using a Nicolet 560 FTIR spectrometer (Nicolet, Waltham, MA). The sizes of SiCWPU dispersions were measured via dynamic light scattering (DLS). The static contact angle of water was measured on the surfaces of films using a DSA30 contact angle system (KRUSS Co.). Dynamic mechanical analysis (DMA) was carried out using a DMA Q-800 dynamic mechanical analyzer (TA Instruments). X-ray photoelectronic spectroscopy (XPS) was performed using an XSAM 800 spectrometer (Kratos, UK). Scanning electronic microscopy (SEM) data from the SiCWPU membranes were measured with a JSM-7500F microscope (model JEOL). The mechanical properties of the SiCWPU films were measured with a material testing machine (5967, Instron, USA).

\section{Synthesis of silicone modified cationic waterborne polyurethane}

Silicone modified cationic waterborne polyurethane (SiCWPU) was synthesized based on a reaction between PPG2000, PDMS and IPDI in a $250 \mathrm{~mL}$ three-necked glass flask fitted with a reflux condenser, a mechanical stirrer and a digital thermometer. $25.0 \mathrm{~g}$ of IPDI, $0.04 \mathrm{~g}$ of DBTDL, and PPG2000 and PDMS at different concentrations (based on the total mass) were added into the glass flask; the reaction proceeded under constant stirring and was kept at $80{ }^{\circ} \mathrm{C}$ until the isocyanate (NCO) content reached the theoretical value. Then the temperature was cooled to $75^{\circ} \mathrm{C}$ and $5.0 \mathrm{~g}$ of MDEA and $4.0 \mathrm{~g}$ of BDO were

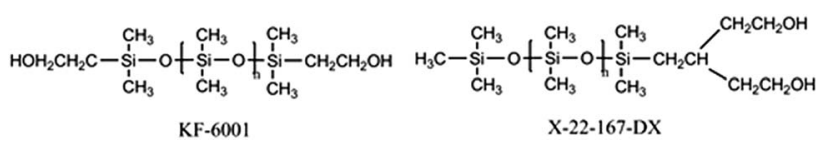

Scheme 1 The chemical structures of polydimethylsiloxane.

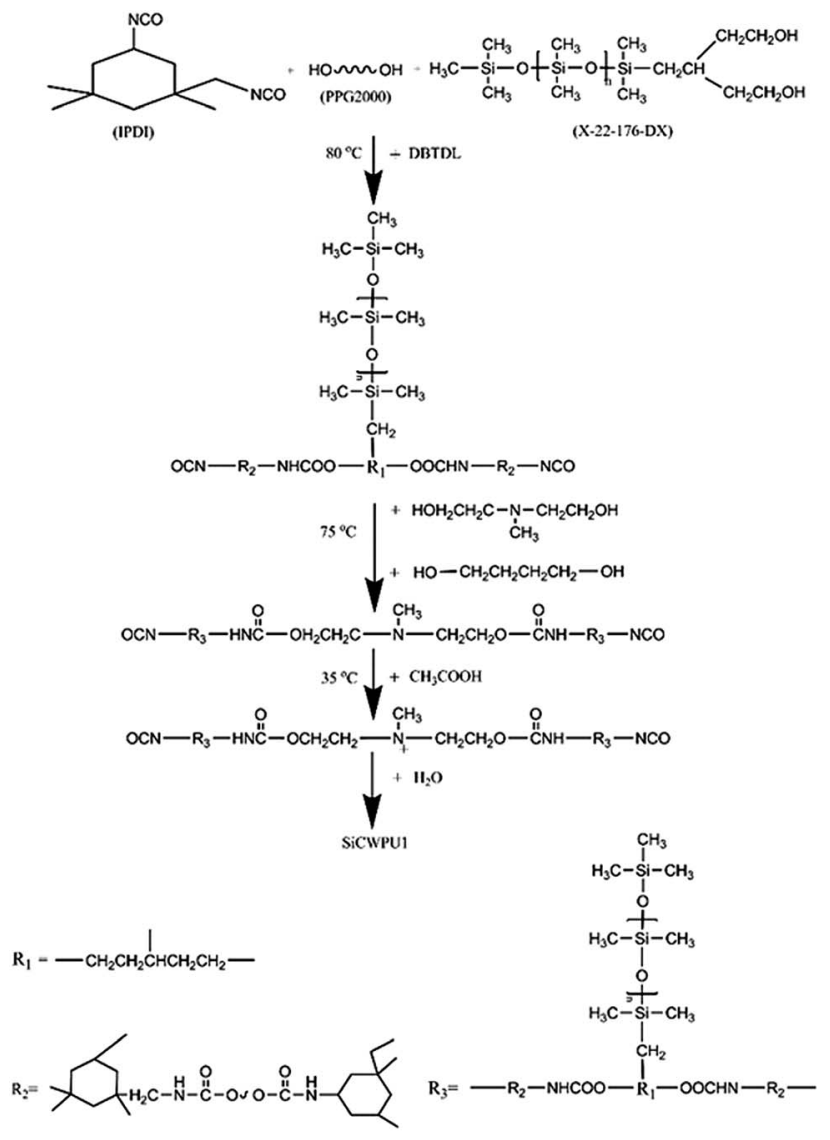

Scheme 2 The synthesis of SiCWPU1 prepared in this study.

slowly introduced to the solution, which was maintained under these conditions for another $2 \mathrm{~h}$. Subsequently, the solution was neutralized using $\mathrm{HAc}$ at $35{ }^{\circ} \mathrm{C}$ for $0.5 \mathrm{~h}$ to obtain the polyurethane prepolymer in the flask. Finally, deionized water was slowly dropped into the solution under vigorous stirring and the solution was maintained under these conditions for an additional $1 \mathrm{~h}$. Finally, the polyurethane prepolymer was rigorously dispersed through being dropped into distilled water under stirring at a speed of $1500 \mathrm{rpm}$ for $1 \mathrm{~h}$ to obtain cationic SiWPU dispersions. The solid content in the SiCWPU samples was $30 \mathrm{wt} \%$. SiCWPU1 was synthesized when the type of PDMS added was X-22-176-DX, and SiCWPU2 was synthesized when KF-6001 was added. The reaction scheme for SiCWPU1 is shown in Scheme 2 and the synthetic formulae are tabulated in Table 1.

\section{Preparation of SiCWPU films}

SiCWPU films were obtained from waterborne polyurethane dispersions on tetrafluoroethylene plates that were allowed to dry at room temperature for 5 days; then the film was dried in an oven at $50{ }^{\circ} \mathrm{C}$ for $24 \mathrm{~h}$. After demolding, the SiCWPU films were kept in a desiccator for vacuum drying preservation.

\section{Fourier-transformed infrared spectroscopy}

Infrared spectra of the SiCWPU films were collected via Fouriertransformed infrared (FTIR) spectroscopy using a Nicolet 560 
Table 1 Compositions of the SiCWPU samples

\begin{tabular}{|c|c|c|c|c|c|c|c|}
\hline Sample & PPG 2000/g & IPDI/g & $\mathrm{MDEA} / \mathrm{g}$ & $\mathrm{BDO} / \mathrm{g}$ & $\mathrm{HAc} / \mathrm{g}$ & KF-6001/\% & $\mathrm{X}-22-176-\mathrm{DX} / \%$ \\
\hline CWPU & 28.8 & 25 & 5.0 & 4.0 & 3.0 & 0 & 0 \\
\hline SiCWPU1-0.5 & 28.7 & 25 & 5.0 & 4.0 & 3.0 & 0 & 0.5 \\
\hline SiCWPU1-1.0 & 28.3 & 25 & 5.0 & 4.0 & 3.0 & 0 & 1.0 \\
\hline SiCWPU1-3.0 & 27.0 & 25 & 5.0 & 4.0 & 3.0 & 0 & 3.0 \\
\hline SiCWPU1-5.0 & 25.8 & 25 & 5.0 & 4.0 & 3.0 & 0 & 5.0 \\
\hline SiCWPU2-0.5 & 28.7 & 25 & 5.0 & 4.0 & 3.0 & 0.5 & 0 \\
\hline SiCWPU2-1.0 & 28.3 & 25 & 5.0 & 4.0 & 3.0 & 1.0 & 0 \\
\hline SiCWPU2-3.0 & 27.0 & 25 & 5.0 & 4.0 & 3.0 & 3.0 & 0 \\
\hline SiCWPU2-5.0 & 25.8 & 25 & 5.0 & 4.0 & 3.0 & 5.0 & 0 \\
\hline
\end{tabular}

FTIR spectrometer (Nicolet, Waltham, MA) at ambient temperature. Thirty-two scans were averaged for the IR measurements over a range of $4000 \mathrm{~cm}^{-1}$ to $600 \mathrm{~cm}^{-1}$, with a resolution of $4 \mathrm{~cm}^{-1}$.

\section{Particle size measurements}

The average particle sizes of the SiCWPU dispersions were analyzed using dynamic light scattering apparatus (LA-960, Horiba, Japan). All samples were diluted to $0.5 \mathrm{wt} \%$ before analyzing the particle size.

\section{Water contact angle assay}

The static contact angle of water was measured on the surface of the films using a DSA30 contact angle system (KRUSS Co.). A 2 $\mu \mathrm{L}$ water droplet was dropped into contact with the surface and the contact angle was reported immediately. The experimental test results were the average values from at least three measurements.

\section{X-ray photoelectron spectroscopy}

X-ray photoelectronic spectroscopy (XPS) was performed using an XSAM 800 spectrometer (Kratos, UK) to analyze the SiCWPU membrane surface element compositions. The exit angle was $90^{\circ}$ and the test depth was $10 \mathrm{~nm}$ from the surface.

\section{Scanning electron microscopy}

Scanning electronic microscopy (SEM) data from the SiCWPU membranes were measured with a JSM-7500F microscope (model: JEOL) operating at an acceleration voltage of $15 \mathrm{kV}$. The samples were sprayed with gold prior to observation. The specimens were magnified 1000 times to detect surface morphology changes.

\section{Water resistance}

$3 \mathrm{~cm} \times 3 \mathrm{~cm}$ SiCWPU films were taken to measure water absorption. The samples were placed in a vacuum oven at $80{ }^{\circ} \mathrm{C}$ for $24 \mathrm{~h}$ and then the weights of the films were measured. The dried SiCWPU films were placed in a Petri dish containing deionized water, and the water absorption of the films was measured after $1 \mathrm{~h}, 2 \mathrm{~h}, 4 \mathrm{~h}, 8 \mathrm{~h}, 12 \mathrm{~h}$ and $24 \mathrm{~h}$. The water absorption $(G \%)$ of the films was calculated using eqn (1).

$$
G /(\%)=\frac{m-m_{0}}{m_{0}} \times 100 \%
$$

where $m_{0}$ is the weight of the dry SiCWPU film and $m$ is the weight of the SiCWPU film after water absorption.

\section{Mechanical properties}

Tensile testing was performed using with a 5967-model material testing machine at a speed of $50 \mathrm{~mm} \mathrm{~min}{ }^{-1}$ and at $25{ }^{\circ} \mathrm{C}$. The dumbbell-shaped specimens used were $25 \mathrm{~mm}$ in length, $4 \mathrm{~mm}$ in width and had a thickness of about $0.5 \mathrm{~mm}$. Analysis of each sample was repeated three times.

\section{Dynamic mechanical analysis}

Dynamic mechanical analysis (DMA) was carried out using a DMA Q-800 dynamic mechanical analyzer (TA Instruments). SiCWPU membranes were tested over a temperature range of $-80{ }^{\circ} \mathrm{C}$ to $100{ }^{\circ} \mathrm{C}$ with a heating rate of $3{ }^{\circ} \mathrm{C} \mathrm{min}^{-1}$.

\section{Results and discussion}

\section{FTIR characterization of the synthesis}

FTIR spectra of SiCWPU1 and SiCWPU2 films are shown in Fig. 1. The characteristic peaks from WPU could be observed in Fig. 1, ranging from $4000 \mathrm{~cm}^{-1}$ to $600 \mathrm{~cm}^{-1}$. The absorption peaks at $3327 \mathrm{~cm}^{-1}$ belong to $\mathrm{N}-\mathrm{H}$ stretching vibrations and the corresponding bending vibration peaks appear at $1531 \mathrm{~cm}^{-1}$. The broad absorption bands at around $1701 \mathrm{~cm}^{-1}$ correspond to $\mathrm{C}=\mathrm{O}$ stretching vibrations. The peaks in the range of $2970 \mathrm{~cm}^{-1}$ to $2875 \mathrm{~cm}^{-1}$ correspond to the $\mathrm{C}-\mathrm{H}$ stretching vibrations of the alkyl chains. These absorption peaks show the presence of urethane in the synthesized CWPUs. The absence of peaks at $2270 \mathrm{~cm}^{-1}$, where the characteristic peaks of $-\mathrm{NCO}$ groups are located, in the spectra confirmed that all isocyanates reacted during the reaction process. In addition, the asymmetric stretching vibration peaks from $\mathrm{Si}-\mathrm{O}-\mathrm{Si}$ and the $\mathrm{C}-\mathrm{O}-\mathrm{C}$ stretching vibration peaks from WPU soft segments were apparent over the wavenumber range from $1200 \mathrm{~cm}^{-1}$ to $1000 \mathrm{~cm}^{-1}$. The peaks at $806 \mathrm{~cm}^{-1}$ were attributed to $\mathrm{Si}-\mathrm{C}$ telescopic vibration peaks. It has been shown that polydimethylsiloxane was successfully introduced into the cationic waterborne polyurethanes. ${ }^{23-25}$ 


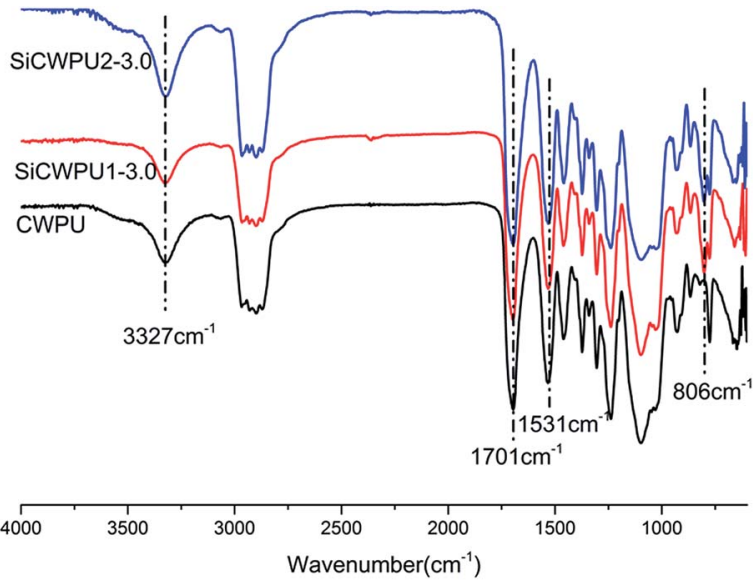

Fig. 1 FT-IR spectra of CWPU, SiCWPU1-3.0 and SiCWPU2-3.0.

\section{Characterization of the water dispersion properties of SiCWPU emulsions}

The size distribution of the CWPU particles is decided by the hydrophilic properties, the chemical structure, the crosslinking, the ionic group content and the chain flexibility. ${ }^{26-28}$ Polysiloxane, as a soft segment, modified cationic aqueous polyurethane, changing the structure of polyurethane and resulting in an increase in the polyurethane particle sizes. ${ }^{29}$ The size distributions of SiCWPU1 emulsions are shown in Fig. 2a. It was observed that the size dispersion of SiCWPU1 particles increased with an increase in the content of X-22-176-DX. The hydrophilic groups and segments in the SiCWPU1 chains tended to migrate to the surfaces of the particles, which was possible as the hydrophobic ingredient X-22-176-DX tended to be packaged in the insides of particles. An increase in the content of X-22-176-DX led to an increase in hydrophobic substance content and a bigger volume in the insides of particles. Therefore, as the organosiloxane content increased, the particle size of the emulsion increased from $37 \mathrm{~nm}$ to $77 \mathrm{~nm}$, as shown in Table 2. The size distributions of the SiCWPU2 particles are shown in Fig. 2b. The reason for the increase in the average particle size was similar to that for SiCWPU1. However, since the X-22-176-DX modified polyurethane formed a comblike structure, the hydrophobic part occupied a larger volume inside the particle as the silicone content increased. Compared with the particle size of SiWPU1, the same content of SiCWPU2 led to a smaller increase in particle size: the specific values are
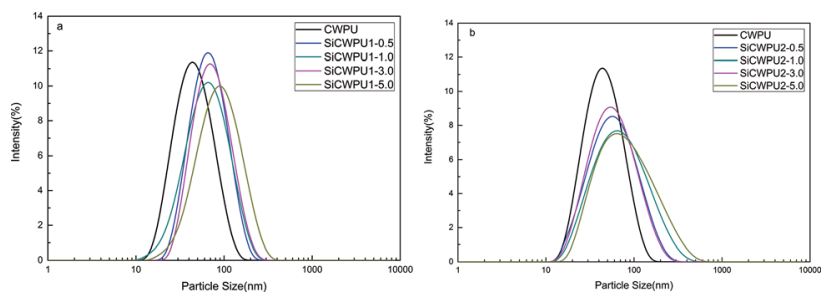

Fig. 2 Particle size distributions of SiCWPU dispersions: (a) SiCWPU1 and (b) SiCWPU2.
Table 2 Particle sizes of SiCWPU1 and SiCWPU2

\begin{tabular}{llll}
\hline Sample & Particle size/nm & Sample & Particle size/nm \\
\hline CWPU & 37.55 & CWPU & 37.55 \\
SiCWPU1-0.5 & 59.12 & SiCWPU2-0.5 & 59.40 \\
SiCWPU1-1.0 & 60.06 & SiCWPU2-1.0 & 59.68 \\
SiCWPU1-3.0 & 66.43 & SiCWPU2-3.0 & 59.92 \\
SiCWPU1-5.0 & 77.63 & SiCWPU2-5.0 & 65.84
\end{tabular}

listed in Table 2. Obviously, the addition of PDMS had an influence on the average particle size of the CWPU dispersions.

\section{X-ray photoelectron spectroscopy}

XPS was used for surface element analysis of the SiCWPU films, which could provide information about the reaction between CWPU and PDMS. XPS survey spectra of SiCWPU1 and SiCWPU2 are presented in Fig. 3. The XPS measurement range for the CWPU sample shows three resolved peaks from carbon $(\mathrm{C} 1 \mathrm{~s}, 285.0 \mathrm{eV})$, nitrogen $(\mathrm{N} 1 \mathrm{~s}, 401.0 \mathrm{eV})$ and oxygen $(\mathrm{O} 1 \mathrm{~s}$, $532.0 \mathrm{eV}) .^{30}$ Comparatively, there were additional peaks from silicon (Si 2s at about 154.0 eV and Si 2p at about $102.0 \mathrm{eV}$ ) following the addition of organosiloxanes, which proved that silicone was incorporated into polyurethane. ${ }^{31}$ Fig. 4 displays a Si 2p XPS survey spectrum from the surface of SiCWPU1-1.0. The peak at $101.6 \mathrm{eV}$ results from the binding energy of Si-O$\mathrm{Si}$ and the peak at $102.1 \mathrm{eV}$ results from the binding energy of $\mathrm{Si}-\mathrm{O}-\mathrm{C}$. The $\mathrm{Si}-\mathrm{O}-\mathrm{Si}$ and $\mathrm{Si}-\mathrm{O}-\mathrm{C}$ bonds were derived from organosiloxane groups that survived during the formation of
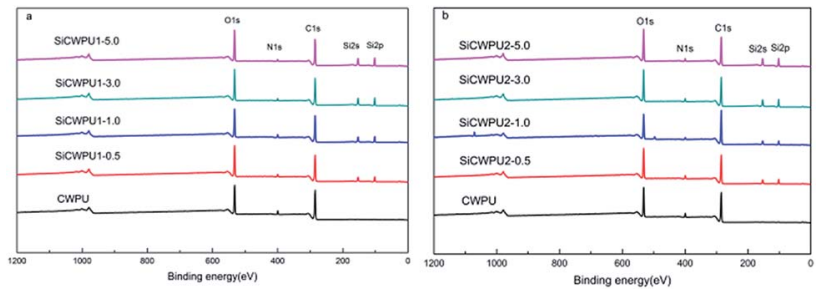

Fig. 3 XPS survey spectra: (a) SiCWPU1 and (b) SiCWPU2.

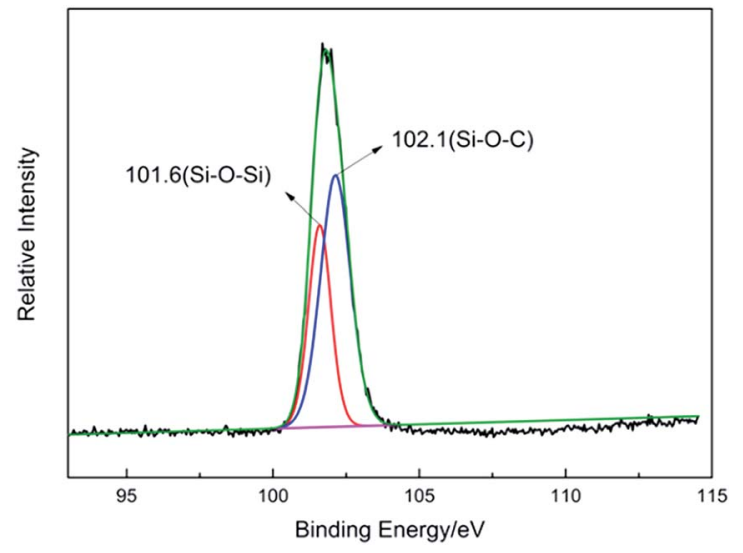

Fig. 4 XPS narrow Si 2p spectrum on the surface of SiCWPU1-1.0. 
Table 3 The surface chemical compositions of SiCWPU1 and SiCWPU2

\begin{tabular}{|c|c|c|c|c|c|}
\hline \multirow[b]{2}{*}{ Sample } & \multicolumn{3}{|c|}{ Concentration/\% } & \multicolumn{2}{|c|}{ Si concentration/\% } \\
\hline & $\mathrm{C}$ & $\mathrm{N}$ & $\mathrm{O}$ & Experimental & Theoretical \\
\hline CWPU & 70.39 & 6.17 & 23.44 & 0 & 0 \\
\hline SiCWPU1-0.5 & 63.27 & 2.70 & 23.10 & 10.94 & 0.04 \\
\hline SiCWPU1-1.0 & 62.72 & 3.22 & 21.24 & 12.82 & 0.36 \\
\hline SiCWPU1-3.0 & 58.79 & 2.22 & 21.72 & 17.28 & 1.80 \\
\hline SiCWPU1-5.0 & 59.55 & 1.81 & 21.72 & 17.92 & 2.52 \\
\hline SiCWPU2-0.5 & 65.81 & 3.45 & 21.79 & 8.95 & 0.04 \\
\hline SiCWPU2-1.0 & 70.49 & 2.08 & 18.43 & 9.00 & 0.36 \\
\hline SiCWPU2-3.0 & 59.69 & 3.10 & 21.71 & 15.50 & 1.80 \\
\hline SiCWPU2-5.0 & 59.15 & 2.63 & 21.78 & 16.45 & 2.52 \\
\hline
\end{tabular}

the film. The Si element percentage on the surface of the film increased with an increase in the PDMS content. The element concentrations on the surfaces of SiCWPU1 films are listed in Table 3. This shows that upon an increase in the X-22-176-DX content, the Si percentage on the film surface increased from 0\% (CWPU) to $17.92 \%$ (SiCWPU1-5.0), which was much higher than the theoretical percentages of $0 \%$ (CWPU) to $2.52 \%$ (SiCWPU1-5.0); the results showed that silicon could migrate to the surfaces of the films. The driving force for migration should derive from the addition of PDMS, making the surface tension of the polymer film lower. ${ }^{32}$

\section{Water contact angle}

Fig. 5 shows the water contact angles of the SiCWPU films. As PDMS was added to the SiCWPU films, the water contact angle increased. SiCWPU1 formed from the modified polyurethane showed an increase in contact angle from $63.0^{\circ}$ (CWPU) to $105.3^{\circ}$ (SiCWPU1-5.0) and tended to stabilize with an increase in X-22-176-DX content. SiCWPU2 formed from the modified polyurethane showed an increase in contact angle from $63.0^{\circ}$ (CWPU) to $100.0^{\circ}$ (SiCWPU2-1.0) and tended to stabilize with an increase in KF-6001 content. This indicates that the SiCWPU films formed a siloxane ( $\mathrm{Si}-\mathrm{O}-\mathrm{Si}$ ) network structure, resulting in a hydrophobic surface. ${ }^{33}$ Moreover, upon adding the same amount of PDMS, the contact angle of SiCWPU1 was higher

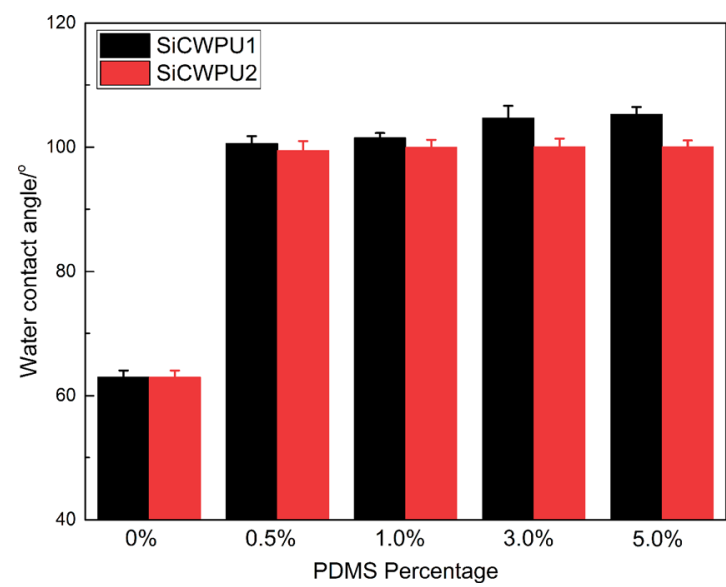

Fig. 5 The water contact angles of SiCWPU films.

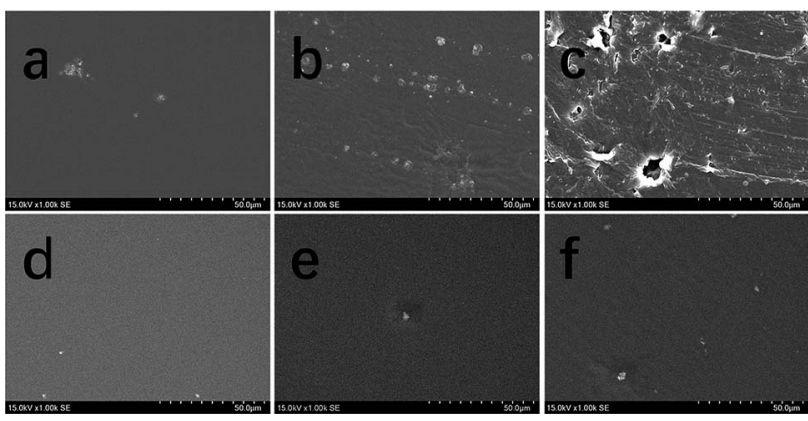

Fig. 6 SEM images of the fracture surfaces of SiCWPU films: (a) SiWPU1-1.0, (b) SiCWPU1-3.0, (c) SiCWPU1-5.0, (d) SiCWPU2-1.0, (e) SiCWPU2-3.0, and (f) SiCWPU2-5.0.

than that of SiCWPU2, which may be because the comb structure formed by SiCWPU1 made it easier for Si to migrate to the surfaces of the films. This was consistent with the results obtained via XPS.

\section{Scanning electron microscopy assays}

The surface morphologies of films with different silica content values, obtained via SEM, are presented in Fig. 6. The test results show that the two different organosiloxane modified cationic waterborne polyurethanes had a certain influence on the surfaces of the polyurethane films, but X-22-176-DX had a greater influence. With the addition of siloxane, white particles appeared on the surfaces of the films, because the introduction of siloxane increased the cross-linking density. ${ }^{29}$ As the PDMS content increased, the molecules tended to agglomerate to form particles. Fig. 6a, d, b, e and c, f show the surface topography analysis of SiCWPU films formed by adding the same amounts of X-22-167-DX and KF-6001 modified cationic waterborne polyurethane, respectively. The results show that adding the same amount of X-22-167-DX was more likely to create larger white particles on the surface of the film than adding KF-6001, and when the X-22-167-DX content was increased by a certain amount, holes were formed on the surface of the film. This may be due to the fact that the comblike structure of SiCWPU1 results in a higher silicon content on the surface of the film.

\section{Water resistance of SiCWPU films}

The water absorption of polyurethane film is related to the hydrophobicity and phase separation of the material. ${ }^{34,35}$ Fig. 7
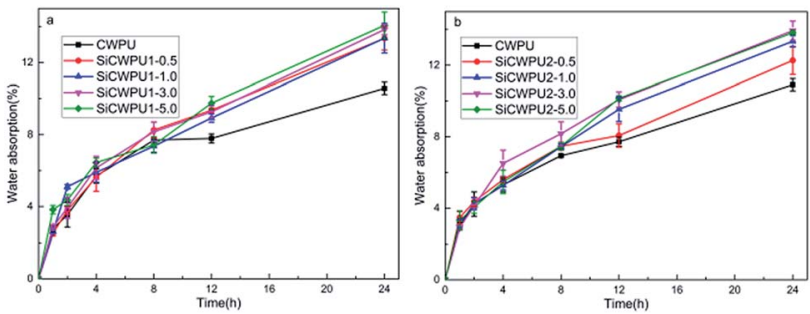

Fig. 7 The water absorption of SiCWPU films: (a) SiCWPU1 and (b) SiCWPU2. 
shows the water absorption of the SiCWPU films. As the PDMS content increases, the water absorption of the polyurethane film increases. After the immersion of SiCWPU films in deionized water for $24 \mathrm{~h}$, the water absorption of the SiCWPU2 films increased from $10.51 \%$ (CWPU) to $14.06 \%$ (SiCWPU2-5.0), and the water absorption of SiCWPU1 films increased from $10.51 \%$ to $13.82 \%$ (SiCWPU1-5.0). This was due to the phase separation of the SiCWPU films, caused by the addition of PDMS. The results showed that the water resistance of the SiCWPU2 films was more affected by phase separation.

\section{Mechanical properties}

Fig. 8 shows stress-strain curves from the SiCWPU films. The results show that the elongation at break of the SiCWPU films increases with an increase in the PDMS content, and the tensile strength decreases. This is due to the fact that PDMS is very soft and has poor tensile strength. ${ }^{36}$ Since SiCWPU1 has a comb-like structure, the silicone segment was more easily stretched in the molecules; the elongation at break of the SiCWPU2 films increased from $617 \%$ to $769 \%$, while the elongation at break of the SiCWPU1 films increased to 959\%. The stress-strain values are listed in Table 4. According to the data in the table, the tensile strengths of the SiCWPU1 films were higher than the SiCWPU2 films when the same amounts of PDMS were added. This may be because the comb-like structure of SiCWPU1 increases the degree of phase separation in SiCWPU1 and increases the tensile strength. The experimental results show that the mechanical properties of SiCWPU1 are better than those of SiCWPU2.

\section{Dynamic mechanical analysis}

Fig. 9 shows the storage modulus values of SiCWPU films as a function of temperature. It is clear that the storage modulus values of the samples at room temperature decrease slowly with an increase in the PDMS content. This was because the addition of PDMS provided flexible Si-O-Si segments in CWPU; a certain degree of microphase separation occurred in the SiCWPU films and the high elastic modulus of the SiCWPU films decreased. ${ }^{37}$

Fig. 10 shows the $\tan \delta$-temperature curves for the SiCWPU films. The peak value of the $\tan \delta$-temperature curve corresponds to the glass transition temperature of the film. It can be seen from the figure that the $\tan \delta$ curves of the films have two peaks, corresponding to the soft transition glass transition temperature $\left(T_{\mathrm{gs}}\right)$ of the CWPU film at $-40.2{ }^{\circ} \mathrm{C}$, and the glass
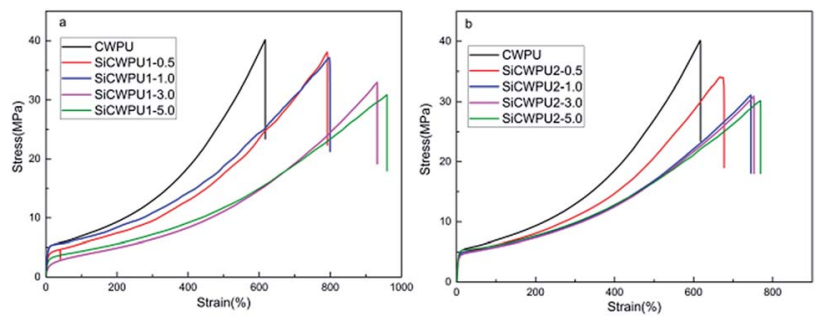

Fig. 8 Stress-strain curves from SiCWPU films: (a) SiCWPU1 and (b) SiCWPU2.
Table 4 Stress-strain data from SiCWPU1 and SiCWPU2 films

\begin{tabular}{lllr}
\hline Sample & $\begin{array}{l}\text { Tensile } \\
\text { strength/MPa }\end{array}$ & $\begin{array}{l}\text { Elongation at } \\
\text { break/\% }\end{array}$ & \multicolumn{1}{l}{$\begin{array}{l}\text { Young's } \\
\text { modulus/MPa }\end{array}$} \\
\hline CWPU & $40.15 \pm 3.15$ & $617 \pm 56$ & $12.36 \pm 2.41$ \\
SiCWPU1-0.5 & $38.06 \pm 4.56$ & $791 \pm 95$ & $8.23 \pm 0.98$ \\
SiCWPU1-1.0 & $37.12 \pm 2.32$ & $799 \pm 67$ & $6.75 \pm 1.25$ \\
SiCWPU1-3.0 & $32.94 \pm 2.56$ & $932 \pm 67$ & $6.60 \pm 1.25$ \\
SiCWPU1-5.0 & $30.89 \pm 3.67$ & $959 \pm 83$ & $4.66 \pm 0.65$ \\
SiCWPU2-0.5 & $34.05 \pm 4.35$ & $677 \pm 45$ & $8.36 \pm 1.32$ \\
SiCWPU2-1.0 & $31.09 \pm 2.78$ & $744 \pm 89$ & $6.50 \pm 2.13$ \\
SiCWPU2-3.0 & $30.79 \pm 5.61$ & $752 \pm 78$ & $6.46 \pm 1.02$ \\
SiCWPU2-5.0 & $30.15 \pm 1.24$ & $769 \pm 67$ & $5.77 \pm 1.54$
\end{tabular}

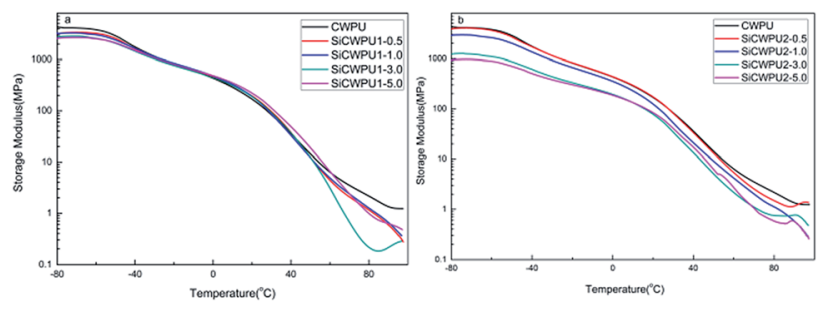

Fig. 9 The storage modulus values of SiCWPU films: (a) SiCWPU1 and (b) SiCWPU2.

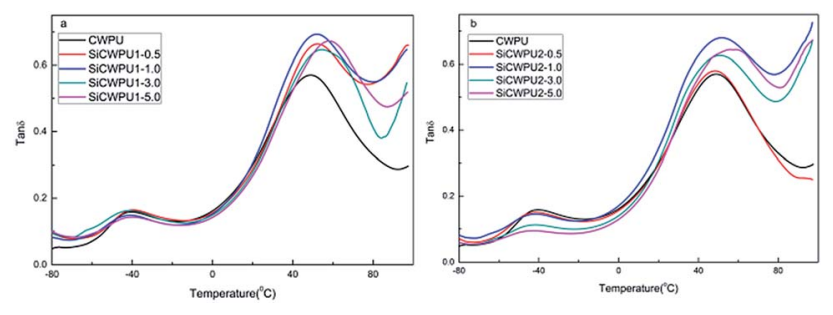

Fig. 10 Tan $\delta$ data from SiCWPU films: (a) SiCWPU1 and (b) SiCWPU2.

transition temperature of the hard segment $\left(T_{\mathrm{gh}}\right)$ of the CWPU at $48.0^{\circ} \mathrm{C}$. The specific values of $T_{\mathrm{g}}$ are listed in Table 5 . According to the data in the table, as the X-22-176-DX content increases, the glass transition temperature of the soft segment did not change substantially, but the glass transition temperature of the hard segment increased from $48.0{ }^{\circ} \mathrm{C}$ to $57.7^{\circ} \mathrm{C}$, indicating that the microphase separation of the film increased upon an increase in the silicon content; the degree of phase separation increased.

Table 5 DMA data from SiCWPU1 and SiCWPU2 films

\begin{tabular}{lcclcc}
\hline Sample & $T_{\mathrm{gs}}{ }^{\circ} \mathrm{C}$ & $T_{\mathrm{gh}} /{ }^{\circ} \mathrm{C}$ & Sample & $T_{\mathrm{gs}} /{ }^{\circ} \mathrm{C}$ & $T_{\mathrm{gh}} /{ }^{\circ} \mathrm{C}$ \\
\hline CWPU & -42.0 & 48.0 & CWPU & -42.0 & 48.0 \\
SiCWPU1-0.5 & -39.7 & 52.0 & SiCWPU2-0.5 & -43.1 & 48.0 \\
SiCWPU1-1.0 & -41.4 & 52.4 & SiCWPU2-1.0 & -42.0 & 50.3 \\
SiCWPU1-3.0 & -41.9 & 54.8 & SiCWPU2-3.0 & -42.0 & 50.9 \\
SiCWPU1-5.0 & -41.4 & 57.7 & SiCWPU2-5.0 & -43.0 & 55.4
\end{tabular}




\section{Conclusions}

A series of SiCWPU films were synthesized using structurally different dihydroxy dimethylpolysiloxane modified cationic waterborne polyurethane examples. The reactions were confirmed through FTIR analysis. KF-6001 and X-22-176-DX were incorporated into CWPU chains with content values increasing from 0.5 to $5.0 \%$, and a series of silane modified CWPU dispersions were prepared using PDMS as the soft segment. The addition of both silanes had a major impact on the properties of the polymers. According to dynamic light scattering characterization, the final particle sizes of the dispersions increased with an increase in PDMS content. However, when the same amount of polysiloxane was added, the resulting SiCWPU1 dispersion was larger than the particle size of the corresponding SiCWPU2 sample. XPS data showed that the strong tendency for the migration of Si elements to the surfaces of the polymer films resulted from the lower surface free energy of silicon. The result was that CWPU coating films exhibited increased surface hydrophobicity. Compared with cationic waterborne polyurethane that was not modified with siloxane, the contact angle following the addition of 5.0\% PDMS was greatly increased. Meanwhile, the contact angle of SiCWPU1 with a siloxane content of $5.0 \%$ was greater than SiCWPU2 with a siloxane content of $5.0 \%$. The stress-strain data indicated that the mechanical properties of SiCWPU1 were better than those of SiCWPU2 when the same amount of PDMS was added. The results from DMA indicated that the glass transition temperature difference between the soft and hard segments increased following the heating of the siloxane, which proved that the addition of polysiloxane increased the phase separation in the polymer film. The results showed that the different structures of silicone modified cationic waterborne polyurethanes resulted in differences in performance. Comblike silicone modified polyurethane performed better than linear silicone modified polyurethane. This study provided an effective route toward CWPU coating materials with advanced application performance.

\section{Conflicts of interest}

There are no conflicts to declare.

\section{Acknowledgements}

This work was funded by the National Natural Science Foundation of China (No. 51773129), the Support Plan of the Science and Technology Department of Sichuan Province, China (2017GZ0129, 2018SZ0174, 2019YFG0257), the International Science and Technology Cooperation Program of Chengdu (2017-GH02-00068-HZ) and the Postdoctoral Research Foundation of Sichuan University (2018SCU12049) and was supported by the Graduate Student's Research and Innovation Fund of Sichuan University (2018YJSY084).

\section{References}

1 W. Sun, Y. Zhou, Y. Ju, L. Yang, T. Xu and Z. Chen, Macromol. Chem. Phys., 2014, 215, 96-102.

2 Y. Fang, J. Li, X. Du, Z. Du, X. Cheng and H. Wang, Polymer, 2018, 158, 166-175.

3 V. J. Tramontano, M. E. Thomas and R. D. Coughlin, in Technology for Waterborne Coatings, ed. J. E.Glass, American Chemical Society, Washington, DC, 1997, pp. 164-182.

4 W. He, Y. Zhang, F. Luo, J. Li, K. Wang, H. Tan and Q. Fu, RSC Adv., 2015, 5, 89763-89770.

5 L. F. Wang, Q. Ji, T. E. Glass, T. C. Ward, J. E. McGrath, M. Muggli, G. Burns and U. Sorathia, Polymer, 2000, 41, 5083-5093.

6 S. Zhang, Z. Chen, M. Guo, H. Bai and X. Liu, Colloids Surf., A, 2015, 468, 1-9.

7 S. Sundar, N. Vijayalakshmi, S. Gupta, R. Rajaram and G. Radhakrishnan, Prog. Org. Coat., 2006, 56, 178-184.

8 Y. Xia, Z. Zhang, M. R. Kessler, B. Brehm-Stecher and R. C. Larock, ChemSusChem, 2012, 5, 2221-2227.

9 N. Sukhawipat, N. Saetung, J.-F. Pilard, S. Bistac and A. Saetung, J. Appl. Polym. Sci., 2018, 135, 45715.

10 A. A. El-Sayed, F. A. Kantouch and A. Kantouch, J. Appl. Polym. Sci., 2011, 121, 777-783.

11 X. Lai, Y. Song and M. Liu, J. Polym. Res., 2013, 20, 1.

12 J. Li, X. Zhang, J. Gooch, W. Sun, H. Wang and K. Wang, Polym. Bull., 2015, 72, 881-895.

13 Y. Song, Y. Gao, X. Wan, F. Luo, J. Li, H. Tan and Q. Fu, RSC Adv., 2016, 6, 17336-17344.

14 F. Zhang, R. Wang, Y. He, W. Lin, Y. Li, Y. Shao, J. Li, M. Ding, F. Luo, H. Tan and Q. Fu, J. Mater. Chem. B, 2018, 6, 4326-4337.

15 R. Wang, F. Zhang, W. Lin, W. Liu, J. Li, F. Luo, Y. Wang and H. Tan, Macromol. Biosci., 2018, 18, e1800054.

16 M.-E. Vlachopoulou, P. S. Petrou, S. E. Kakabakos, A. Tserepi, K. Beltsios and E. Gogolides, Microelectron. Eng., 2009, 86, 1321-1324.

17 X. Z. Kong, D. Zou and X. Zhu, J. Polym. Res., 2014, 21, 8537.

18 H. Wang, Y. Shen, G. Fei, X. Li and Y. Liang, J. Colloid Interface Sci., 2008, 324, 36-41.

19 F. Yu, L. Cao, Z. Meng, N. Lin and X. Y. Liu, Polym. Chem., 2016, 7, 3913-3922.

20 J. Huang, S. Wang, W. Sun, Z. Zhang, C. Cheng, Y. Ju, P. Yang, L. Ding and Z.-R. Chen, Macromol. Chem. Phys., 2015, 216, 2279-2286.

21 C. Xu, L. OuYang, H. Liu, Q. Chen, Z. Cai, J. Xing and Y. Li, Text. Res. J., 2015, 85, 2040-2050.

22 X. Li, G. Fei and H. Wang, J. Appl. Polym. Sci., 2006, 100, 4046.

23 S. Du, Y. Wang, C. Zhang, X. Deng, X. Luo, Y. Fu and Y. Liu, J. Mater. Sci., 2018, 53, 215-229.

24 M. Li, F. Liu, Y. Li and X. Qiang, $R S C A d v ., 2017$, 7, 1331213324.

25 J. Xiao, Z. Qiu, W. Yang, J. Qiu, T. Yang, Y. Xu, Y. Zeng, F. Wang and S. Li, Prog. Org. Coat., 2018, 116, 1-6. 
26 V. García-Pacios, J. A. Jofre-Reche, V. Costa, M. Colera and J. M. Martín-Martínez, Prog. Org. Coat., 2013, 76, 1484-1493.

27 S. K. Lee and B. K. Kim, J. Colloid Interface Sci., 2009, 336, 208-214.

28 S. Wang, Z. Du, X. Cheng, Y. Liu and H. Wang, J. Appl. Polym. Sci., 2018, 135, 46093.

29 C. Fu, X. Hu, Z. Yang, L. Shen and Z. Zheng, Prog. Org. Coat., 2015, 84, 18-27.

30 J. Zhao, T. Zhou, J. Zhang, H. Chen, C. Yuan, W. Zhang and A. Zhang, Ind. Eng. Chem. Res., 2014, 53, 19257-19264.

31 S. Wu, J. Wang, J. Shao, L. Wei, K. Yang and H. Ren, ACS Appl. Mater. Interfaces, 2017, 9, 28887-28901.
32 Q. Li, L. Guo, T. Qiu, W. Xiao, D. Du and X. Li, Appl. Surf. Sci., 2016, 377, 66-74.

33 M. Yeh, C. Yao, C. Hsieh, H. Yang and C.-P. Wu, Eur. Polym. J., 2008, 44, 2777-2783.

34 S. Zhang, Z. Chen, M. Guo, J. Zhao and X. Liu, RSC Adv., 2014, 4, 30938.

35 X. Z. Kong, D. Zou and X. Zhu, J. Polym. Res., 2014, 21, 8537.

36 F. Yu, X. Xu, N. Lin and X. Y. Liu, RSC Adv., 2015, 5, 7254472552.

37 Z. Wu, H. Wang, X. Tian, P. Cui, X. Ding and X. Ye, Phys. Chem. Chem. Phys., 2014, 16, 6787-6794. 